\title{
Rolling resistance of a spherical pod on a granular bed
}

\author{
Stefaan Van wal · Simon Tardivel · Paul Sánchez • Darius \\ Djafari-Rouhani · Daniel Scheeres
}

Received: date / Accepted: date

\begin{abstract}
The motion of a spherical ball rolling without slipping or plowing on a granular bed is studied. We propose a definition of the rolling resistance force and torque, and carry out experiments with a basketball and medicine ball rolling on a bed of gravel to measure the corresponding rolling resistance coefficients. These experiments reveal, in good agreement with literature, coefficients that are velocity-independent, and show that little to no plowing of the spheres into the granular substrate occurs. This indicates a regime of motion distinctly different from those treated in previous works. A simplified model correctly predicts the velocity independence and suggests an inverse dependence of the rolling resistance coefficient on the reduced inertia of the ball. These predictions match the experimentally observed behavior. Numerical simulations based on soft-sphere DEM shed more light on the mechanics of energy dissipation that occur in this no-plowing regime, and reveal a mass dependency that is not captured by the model. Our results provide insight into an unstudied regime of motion, and are of interest to the mission design of spacecraft to explore the surfaces of asteroids and comets.
\end{abstract}

PACS 81.05.Rm, 45.50.Dd, 95.55.Pe, 96.30.Ys

Keywords granular, rolling, resistance, friction

\section{Introduction}

The deployment of instrumentation packages to the surfaces of asteroids and comets can be achieved with low-

S. Van wal

Celestial and Spaceflight Mechanics Laboratory

University of Colorado Boulder

431 UCB, Boulder, CO 80309

E-mail: stefaan.vanwal@colorado.edu risk, low-cost, spherical lander pods that ballistically descend to the surface and settle following a number of bounces and a period of rolling motion [1,2]. To enable development of appropriate pod and mission designs, it is necessary to characterize this rolling motion with realistic models of the interaction between a pod and the asteroid surface, in the microgravity environment of the asteroid.

Numerous studies have analyzed the motion of small spheres, with sizes ranging between a few millimeters and a few centimeters, rolling on a thin granular bed; these studies are briefly summarized here. Rolling spheres experience a force that reduces their rotation and velocity, called rolling friction or rolling resistance [3]. The set-up where a single layer of grains is glued to an inclined plane, a situation in which plastic deformation and grain-grain interactions do not occur, has been frequently documented. Centimeter-sized spheres rolling on such inclined beds of sand demonstrate three regimes of motion, each occurring at different ranges of the angle of incline $4,5,6,7,8,9]$. At intermediate-tohigh inclinations, the rolling resistance force behaves viscous-like and is strongly dependent on velocity [10. In contrast, at small angles of inclination, the force is found to be constant for small spheres rolling on sand at velocities higher than $0.2 \mathrm{~m} / \mathrm{s}$ [11. This friction force allows for the definition of a coefficient of rolling friction, in analogy with the coefficient of sliding friction, as done by [12], where its value is developed analytically for a viscous sphere rolling on a hard, flat plane.

Comparatively few works have investigated the motion of a sphere rolling on a loosely packed bed of sand, where individual grains are free to move and interact with each other. Experiments with centimetersized glass and steel spheres rolling on an inclined sand bed again revealed a constant coefficient of rolling re- 
sistance, between 0.45 and 0.65 for the various spheres tested [3]. In this configuration, the spheres' plowing into the sand was found to be the main mechanism for energy dissipation and the source of the observed rolling resistance force. This was shown through a measurement of the depth of the sand grooves created by the rolling spheres, which reach several millimeters for centimeter-sized spheres. The authors of [3] suggest an extension of their work to the motion of larger spheres on similar granular beds.

This paper covers the regime of motion of such larger spheres, which is observed to be differentiated from that of smaller spheres through their relative levels of plowing. We carry out experiments at $1 g$ with spheres roughly the size of pods proposed for small-body missions, measure the magnitude of their rolling resistance coefficients, and examine the spheres' level of plowing into the granular substrate. Concurrently, a simple model is developed, based on the dynamics of contact between the sphere and the grains in the absence of plowing. Finally, numerical simulations are used to gain further insight into the motion of the spheres and the effect of their masses. Using this combination of experiment, theory, and simulation, we provide insight into what factors govern contact dynamics under the assumption of no plowing, and provide an order-ofmagnitude estimation of the rolling resistance coefficients. Our results extend the existing knowledge on the mechanics of rolling resistance by probing a previously unstudied regime of motion. They are relevant to geophysical applications and simulations of lander trajectories to the small bodies of our Solar System, such as asteroids, comets, and small moons.

\section{Rolling Resistance}

A sphere rolling on a granular bed will experience a dissipative force that resists its rolling motion. We postulate that this phenomenon can be captured by applying a force $F_{r r}$ and torque $L_{r r}$, called the rolling resistance, to a ball rolling on a perfectly flat surface. $F_{r r}$ is applied at the center of the ball and directed against the velocity; $L_{r r}$ is directed against the angular velocity. We denote $R$ the radius of the ball, $I$ its massless inertia and $N$ the normal force from the surface $(N=m g)$. The magnitudes of the rolling resistance quantities are defined as:

$F_{r r}=K_{r r} N \quad$ and $\quad L_{r r}=C_{r r} R N$

where $C_{r r}$ and $K_{r r}$, related through $C_{r r}=j K_{r r}$ with $j=I / R^{2}$, are defined $a d h o c$ and are called the coefficients of rolling resistance. This relationship must hold in order for rolling resistance to preserve the slip state of the sphere, which is determined by the (Coulomb) friction force and torque. The quantity $j$ is dimensionless and represents the mass distribution of the ball relative to its center. The value $m R^{2}$ represents the maximum inertia that can be reached by an object of maximum radius $R$. It is in fact attained by the circle of radius $R$. The quantity $j$ varies between 0 (a ball whose mass is concentrated at the center) and 1 (a hoop). For a sphere of homogeneous density, $j=2 / 5$, and for a spherical shell, $j=2 / 3[13$. Note that $j \in[0,1]$ implies that $C_{r r} \leq K_{r r}$.

The quantity $(1+j)$ is of further interest, and manifests in the specific kinetic energy $\mathcal{E}$ of a ball rolling at velocity $V$ without slip, as: $\mathcal{E}=0.5(1+j) V^{2}$. Applying a force $F$ to the center of a ball or a wheel rolling without slip does not create an acceleration equal to $F / m$. When rolling without slip is enforced, the friction force partly counters the force $F$. In fact, the force $F$ creates an acceleration equal to $F /(1+j) m$. Remembering that inertia is defined as the resistance an object has to a change in its state of motion, then $(1+j) m$ is the effective inertia of the ball, i.e. the resistance to change in its motion. We therefore expect $K_{r r}$ to depend strongly the quantity $(1+j)$, which is seen as the reduced inertia of the ball, while $j$ is interpreted as its reduced moment of inertia.

\section{Experiments}

Spherical lander pods have previously been proposed for the Binary Asteroid in-situ Explorer (BASiX) space mission, and are roughly the size of a basketball [1,2]. With this hardware description in mind, we have chosen to use both a standard basketball and a similar-sized, heavier medicine ball, and experimentally measure their rolling resistance coefficients. The basketball has a radius of $R \approx 119 \mathrm{~mm}$ and a mass of $m \approx 0.63 \mathrm{~kg}$; the medicine ball has a radius of $R \approx 113 \mathrm{~mm}$ and a mass of $m \approx 2.70 \mathrm{~kg}$. We obtain measurements of the coefficient of rolling resistance by rolling these balls on a bed of gravel, tracking their motion with a high-speed camera, and processing the obtained video material with a vision algorithm.

The granular bed consists of pea gravel with grain sizes between $6.4 \mathrm{~mm}$ and $12.4 \mathrm{~mm}$ in size, assumed to be distributed uniformly. This gravel was measured to have a grain density of $\rho_{\text {grain }}=2.62 \mathrm{~g} / \mathrm{cm}^{3}$ and a bulk density of $\rho_{\text {bulk }}=1.52 \mathrm{~g} / \mathrm{cm}^{3}$, corresponding to a filling fraction of 0.58 and a porosity of 0.42 . Note that these ratios are only valid for uncompacted and unshaken grain samples. Finally, the angle of friction was measured to be $\approx 41^{\circ}$. 


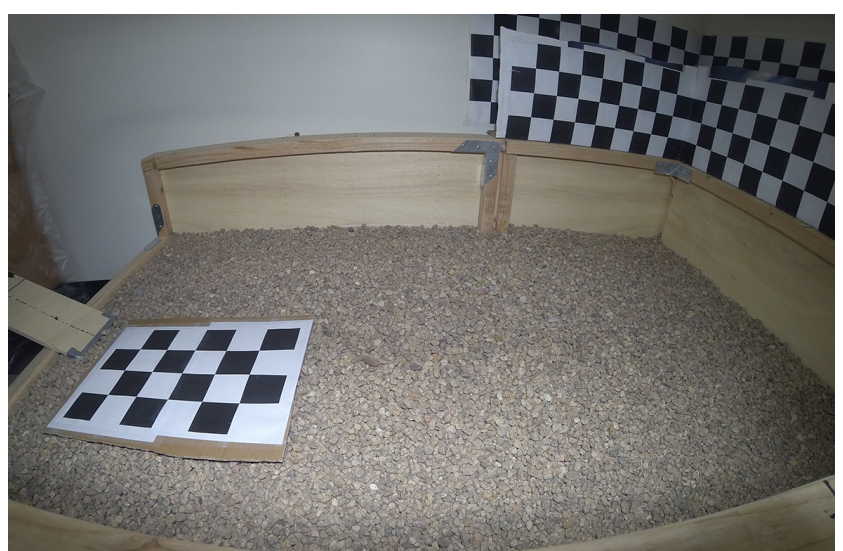

Fig. 1 Camera distortion and calibration patterns.

The gravel was contained in a wooden box $(1.6 \mathrm{~m} \times$ $1.0 \mathrm{~m} \times 0.5 \mathrm{~m})$; a ramp placed on one end of the box was used to launch the balls from rest along the box's longest dimension ( $x$-axis) with consistent specific energy. The motion of the balls was recorded using a GoPro HERO3 Black Edition camera, which has a wideangle fish-eye lens capable of capturing video at high resolution and frame-rate. The camera was mounted on a tripod and positioned above the center of the longest side of the box, providing an optimal overview of the entire scene. Recording was set to a $1280 \times 960 \mathrm{px}$ resolution, $100 \mathrm{fps}$ frame-rate, and $127^{\circ}$ field-of-view. Moreover, a floodlight was mounted directly above the camera to minimize shadowing, together with a wooden casing protecting the camera from the floodlight's heat. Finally, both balls were spray-painted bright green to facilitate detection in the video analysis, as discussed further on.

Prior to running the experiments, the camera is positioned in the desired location and parallel with the local horizontal. It is then switched on and connected to a mobile device using the GoPro App, removing the need for the user to physically interact with the camera and ensuring its position remains unchanged throughout the entire batch of experiments. The wooden casing and floodlight are placed around the camera once it has been switched on. Afterwards, the camera captures several photos of checkerboard patterns, sequentially placed at different positions and orientations in the field of view of the camera. These photos are to be used for calibration purposes and the definition of a reference frame, prior to processing the video recordings, as will be discussed shortly.

The gravel surface is then flattened to the best degree possible using a flat wooden board, so as to minimize any bias acceleration due to an inclined surface. This flattening compacts the grains, thereby affecting the magnitude of the rolling resistance. The grains are therefore decompressed by gently sweeping a broom across the surface. This returns the surface to its initial rough and uncompacted state, while still ensuring it remains flat and level.

In each experimental run, the ball is released from the same point along the ramp using a restraint, to ensure a consistent initial velocity on the gravel bed. The camera starts recording, the ball is released, and starts rolling. It accelerates down the ramp and continues rolling across the gravel surface, decelerating under the influence of the rolling resistance force and torque until it comes to a halt near the end of the box, completing one experimental run. As the ball compacts some of the grains along its path on the gravel, the surface must be swept with a broom after each single run to ensure proper surface roughness. These procedures are consistent with those applied for experiments on mobile beds in previous work [3]. In addition, we also re-flatten the surface every 5 runs. This strategy is repeated 50 times to obtain a single video batch; two such batches are recorded for each ball, yielding a total of 200 recorded videos.

Before proceeding with an analysis of the video material, the camera calibration parameters are first obtained using the previously captured photos and Matlab's built-in calibration tool. This calibration is necessary to account for the strong image distortion that occurs because of the fish-eye lens of the GoPro, as shown in Fig. 1.

The recorded video material is then analyzed through a Matlab algorithm. This algorithm first applies a selective grayscale filtering which weighs particular colors more strongly than others, contrary to a 'regular' grayscale image in which all colors are weighted equally. It was found that bright green objects are most easily detected by the algorithm, motivating our choice to spray-paint the balls in this color. A sample frame with such selective grayscale applied is shown in Fig. 2 .

Next, edge detection is applied to the grayscale frames to render visible only the edges of any distinct objects or faces in the scene, through a detection of sudden changes in the intensity between neighboring pixels. Finally, a Hough transform is applied to fit circles to the resulting edge-detected frames, as the target ball will be the largest spherical object present in each frame. With the angular and pixel resolution given, the maximum position error for each individual pixel is $1.2 \mathrm{~cm}$ when the camera is placed at a nominal distance of 1 $\mathrm{m}$ from the ball. As we assume the error distribution to be Gaussian, the circle fitting applied by the Hough transform effectively averages this error along the edge of the ball. The resulting position error of the center of the ball is therefore reduced by over an order of magn- 


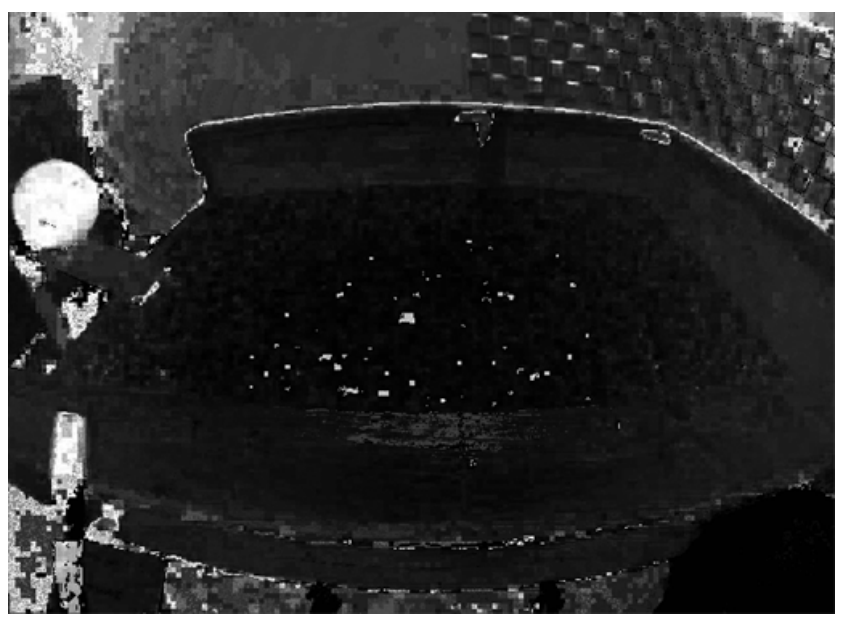

Fig. 2 Video frame filtered with selective grayscale. The ball is visible on the left.
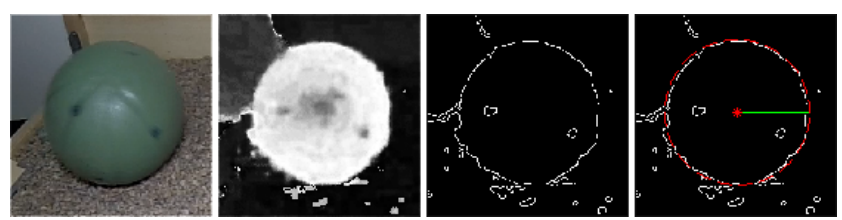

Fig. 3 Vision algorithm steps applied in tracking of sphere.

tiude. By applying these three steps; selective grayscale, edge detection, and circle fitting, the position of the ball can be extracted from each video frame. The sequential application of these three steps to the area of interest in a sample frame is shown in Fig. 3.

By processing an experiment video with this vision algorithm, we obtain the tracked 'raw' trajectory of the ball across the two-dimensional view of the camera, as shown in Fig. 4. Through a series of transformations using the previously obtained camera calibration parameters and the known ball radius, we can account for the fish-eye effect, apply undistortion to the coordinates of the ball, and obtain a Cartesian representation of its three-dimensional trajectory, where the ball moves along the $x$-direction of the applied reference frame.

It has been found both experimentally and theoretically in various works that the motion of a sphere on a granular bed can be affected by constant rolling resistance forces [3, 11, 12, as well as viscous rolling resistance forces 10. As it is visually clear that the spheres did not significantly plow into the granular bed, we do not anticipate significant viscous forces. Nonetheless, we allow for the existence of both constant and viscous terms to exist, as both are encountered in the literature, and assume the force to be quadratically dependent on velocity. This allows us to investigate the possible existence of viscous terms in a mathematically rigorous manner. Noting $x$ and $\dot{x}$ as respectively the position and velocity along the $x$-axis, and $a, b$, and $c$ as con-

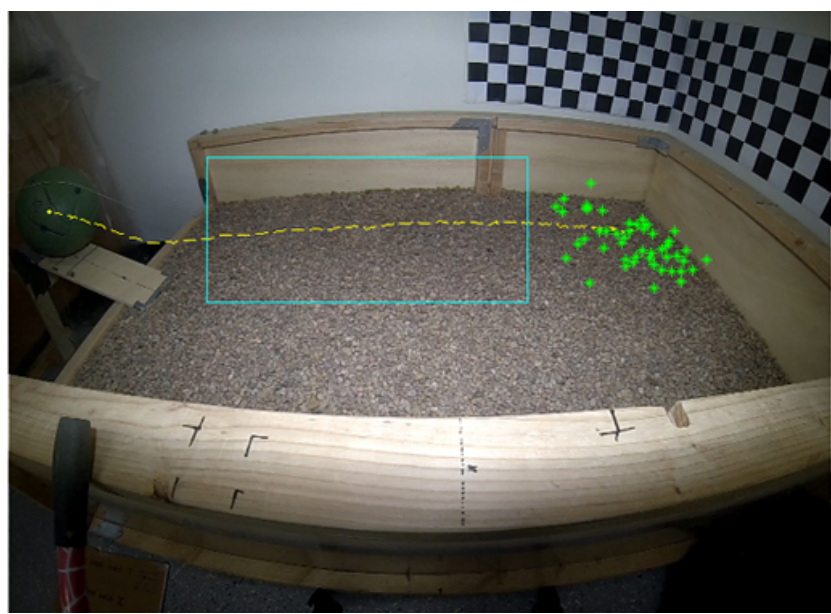

Fig. 4 Tracked trajectory of a single experiment. The green crosses are the final positions of previously analyzed videos.

stant positive coefficients, the motion of the balls was hence assumed to follow the equation:

$\ddot{x}=-\left(a+b \dot{x}+c \dot{x}^{2}\right)$

This model captures dependencies on the velocity through the inclusion of the $b$ and $c$ terms. This differential equation is an integral form of Ricatti's differential equation, with the initial condition $\dot{x}(0)=V_{0}$. Its solution can be expressed analytically as:

$x=\frac{1}{c} \ln \left[\cos \left(\frac{1}{2} \delta t-\phi\right)\right]-\frac{1}{c} \ln [\cos (\phi)]-\gamma t$

where $\delta=\sqrt{4 a c-b^{2}} ; \kappa=\frac{\delta}{2 c} \quad ; \quad \gamma=\frac{b}{2 c}$

and $\phi=\arctan \left(\frac{V_{0}+\gamma}{\kappa}\right)$

Please note that the previous equation does not place constraints on the reals $a, b$, and $c$. In particular, they do not require that $4 a c-b^{2}>0$ as long as one considers the functions presented above (square root, logarithm, and trigonometric functions) with their natural complex extensions. Moreover, singularities can be resolved by taking limits.

The extracted $x$-position data of the ball was fit to Eq. 3 using a non-linear batch least squares estimator, applied to all 100 videos of each ball. This produced negligible $b$ and $c$ values close to machine precision level $\left(\approx 10^{-12}\right)$, experimentally confirming our intuition and the results of 3, 11] that, for this regime, the rolling resistance force is independent of velocity. Furthermore, this lack of velocity-dependent terms indicates that aerodynamic drag did not have a notable effect on the motion of the balls. Taking the limit of $c \rightarrow 0$ and $b \rightarrow 0$, the complex expression of Eq. 2 simplifies to the classic constant deceleration motion:

$x=v_{0} t-\frac{1}{2} a t^{2}$ 
A simple second-order polynomial fit on the position of the ball during this deceleration then yields the value of the coefficient $a$, and thus of $K_{r r}=a / g$, as defined through Eq. 1. We note that, although the balls travel purely along the $x$-axis for most of the experiment duration, they display some deviation perpendicular to the direction of travel near the end of each run. For this reason, the final portion of the trajectories (where $V<0.1 \mathrm{~m} / \mathrm{s}$ ) are excluded from our fitting; the included portion is marked by a cyan box in Fig. 4. This results in a mean $K_{r r}=0.0524$ with a standard deviation $\sigma=0.0067$ for the basketball, and a mean $K_{r r}=0.0655$ with $\sigma=0.0116$ for the medicine ball. Given that both balls have similar radii, are made of similar material, and roll on the same granular substrate, the difference between the two $K_{r r}$ must be due to the differing mass $m$ and inertia $j$ of the two balls.

Comparing the values of these $K_{r r}$ to those measured for the motion of the centimeter-sized spheres rolling on an inclined sand bed where $K_{r r}=[0.45,0.65]$ [3], we find that the values obtained in our experiments are an order of magnitude lower. The authors of 3 measured the groove left behind by a glass sphere with radius $R=14.2 \mathrm{~mm}$ rolling on sand as $h \approx 4 \mathrm{~mm}$, corresponding to the significant plowing ratio of $\eta_{g s}=$ $h / R \approx 0.28$. Measuring the grooves in our experiments, we have found depths in the gravel of $h_{b b} \approx 1 \mathrm{~mm}$ for the basketball and $h_{m b} \approx 3 \mathrm{~mm}$ for the medicine ball, resulting in comparatively low plowing ratios of respectively $\eta_{b b}=0.01$ and $\eta_{m b}=0.03$. This matches the visually observed absence of plowing in our considered regime of motion, and appears to be the source of the (relatively) low magnitude $K_{r r}$.

In conclusion, these experiments indicate a velocityindependent coefficient of rolling resistance for large spheres rolling on an a granular surface without plowing. Given the similar sphere radii, the differences in measured coefficient magnitudes must be due to the spheres' different mass and inertia. These dependencies invite further study, and are investigated in the remainder of this work.

\section{A Simple Model}

In parallel with the experiments discussed above, we developed a simple model that attempts to capture some of the observed trends. As early experimental results showed that both balls displayed little to no plowing into the substrate, we develop this model based on the no-plowing assumption. In other words, the rolling resistance force experienced in this regime can be estimated with the assumption that grains in the substrate do not move as the sphere rolls across the granular bed.
In a coarse approximation, the bed may thus be modeled as a succession of collisions on a rigid surface with very small asperities. When a ball, rolling without slipping, impacts a small rock, it dissipates energy through two collisions occurring in rapid succession. First, the ball impacts the rock and the velocity normal to this impact is damped. At the same time, the friction force transfers part of the angular momentum into linear momentum, increasing slightly the velocity of the ball, but diminishing slightly its angular velocity, and dissipating energy in the process. The ball is then launched into a short ballistic arc. Second, the normal velocity is damped again when it re-impacts the ground, and the friction force once again synchronizes the body spin and velocity, returning it to its initial state but with a reduced velocity and rotation rate. Fig. 5 depicts these events.

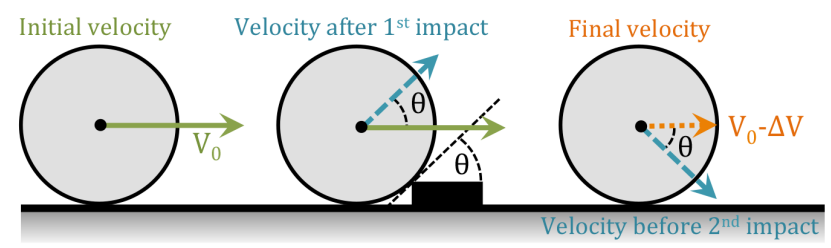

Fig. 5 Micro-collision model of a sphere rolling on a granular bed. The size of the granular bed and particle have been exaggerated for clarity of the drawing.

The value of the coefficient $K_{r r}$ can be established using the micro-collision approach introduced. The two collisions are assumed to take place with a null coefficient of restitution (whose influence is otherwise negligible at first order for small collision angles $\theta$ ) and an infinite coefficient of friction (to ensure no-slip conditions throughout the motion). Let $V_{0}$ be the initial speed of the ball and $d$ the height of the feature being impacted, then the total loss of speed $\Delta V$ is:

$\Delta V=V_{0}\left(1-\zeta(\theta)^{2}\right)$

where $\zeta(\theta)=\frac{j+\cos \theta}{j+1}$

and $\theta=\arccos \left(\frac{R-d}{R}\right)$

Assuming that micro-collisions are so frequent that one occurs right after the ball has re-impacted the ground, the time between two events is:

$\Delta t=\frac{2 V_{0} \zeta(\theta) \sin \theta}{g}$

Dividing Eq. 5 by Eq. 6 and normalizing by $g$, we obtain:

$K_{r r}=\frac{1}{g} \frac{\Delta V}{\Delta t}=\frac{1-\zeta(\theta)^{2}}{2 \zeta(\theta) \sin \theta}$ 
Taking $d / R \ll 1$, as the radius of the ball is much larger than the size of the impact features, Eq. 7 simplifies to:

$K_{r r} \approx \frac{1}{1+j} \sqrt{\frac{d}{2 R}}$

The value of $d$ should be the average difference of height between successive grains of the gravel bed. For a uniform size distribution of range $\left[d_{\min }, d_{\max }\right]$, this average difference is $d=1 / 3\left(d_{\max }-d_{\min }\right)$. Using the numbers provided by the gravel manufacturer, the ratio $d / R$ was estimated to be close to 0.0166 . For this configuration, the analytical model predicts a value of $K_{r r}=0.055$ for a spherical shell, and $K_{r r}=0.066$ for a solid sphere.

This very simple expression predicts some key results that shed light on the experimental observations. Most importantly, it predicts a rolling resistance coefficient which is independent of the ball velocity. This matches the observed behavior and agrees with literature 3. Second, the model predicts that in the considered regime, any variation in $K_{r r}$ comes purely from variations in the reduced inertia $(1+j)$. This predicted variation is in very good agreement with the observed values; indeed, the predicted and observed coefficients match almost exactly. Given the simplicity of the model, it is likely that the exact match is coincidental, though it remains that the model predicts a correct order of magnitude and the observed dependencies. The inverse dependency on the reduced inertia $(1+j)$ makes intuitive sense, as this quantity represents the resistance of a ball to change in its rolling motion.

Although the observed trends appear to match the inertia dependency, the model does not suggest any dependency on the ball mass or the properties of the granular substrate. This is likely the result of the noplowing assumption, and illustrates the limitations of this simple model. In order to gain further insight into the experiments and potential mass dependencies, we will continue our investigation using simulations.

Additionally, we may compute the ratio of rolling resistance coefficients of respectively the experimental data and the model. Using the measured coefficients, we find a ratio of:

$$
\left(\frac{K_{r r, m b}}{K_{r r, b b}}\right)_{e x p}=1.25
$$

When instead using Eq. 8 to compute this ratio, we predict:

$$
\begin{gathered}
\left(\frac{K_{r r, m b}}{K_{r r, b b}}\right)_{m o d}=\frac{\frac{1}{1+j_{m b}} \sqrt{\frac{d}{2 R_{m b}}}}{\frac{1}{1+j_{b b}} \sqrt{\frac{d}{2 R_{b b}}}} \\
=\frac{1+j_{m b}}{1+j_{b b}} \sqrt{\frac{R_{m b}}{R_{b b}}}=1.22
\end{gathered}
$$

This shows that the predicted coefficient ratio is independent on the grain height $d$, and matches the measured ratio to within $2.5 \%$. This further strengthens the agreement between the model prediction and the experiments.

On a final note, we see that Eq. 8 is also independent of the gravitational acceleration $g$, which allows for our model to be applied to the microgravity environment present on the small bodies of our Solar System. The time required for a ball to come to rest under the influence of rolling resistance can be found as $t=V_{0} /\left(K_{r r} g\right)$, which does depend on the gravitational acceleration. As an example, we compute this time on asteroid Itokawa, which has a surface gravitational acceleration of $g \sim 10^{-5} \mathrm{~m} / \mathrm{s}$. The minimum velocity at which continued contact is possible is roughly $V_{0} \sim 8 \mathrm{~mm} / \mathrm{s}$ [14, such that we find a settling time of $t \sim 3.3 \mathrm{hr}$ for a solid sphere. In contrast, a solid sphere rolling with an initial velocity of $V_{0}=1 \mathrm{~m} / \mathrm{s}$ on Earth (where $g=9.81 \mathrm{~m} / \mathrm{s}^{2}$ ) has a settling time of only $t=1.5 \mathrm{~s}$, in agreement with our experiments.

\section{Simulations}

We carry out numerical simulations using a computational code that implements a soft-sphere DEM [15,16. 17, 18, 19, 20, 21. In this method, the bed particles are modeled as spheres that follow a predetermined size distribution, can shift around within the bed, and interact through a soft-repulsive potential when in contact. When two particles overlap, they are considered to be in contact. When this happens, normal and tangential contact forces are calculated 22 . The former is modelled by a linear spring-dashpot system and is always repulsive, keeping the particles apart; the latter is also modelled with a linear spring that satisfies the local Coulomb yield criterion. The calculation of the normal forces between colliding particles is modeled by a linear spring and a dashpot. The elastic force is modelled as:

$\mathbf{f}_{e}=k_{n} \xi \hat{\mathbf{n}}$,

Similarly, the damping force is modeled as:

$\mathbf{f}_{d}=-\gamma_{n} \dot{\xi} \hat{\mathbf{n}}$

The total normal force is calculated from these as $\mathbf{f}_{n}=$ $\mathbf{f}_{e}+\mathbf{f}_{d}$. In these equations, $k_{n}$ is the elastic constant, $\xi$ is the overlap of the particles, $\gamma_{n}$ is the damping constant (related to the dashpot), $\dot{\xi}$ is the rate of deformation, and $\hat{\mathbf{n}}$ is the vector joining the centres of the colliding particles. This dashpot models the energy dissipation that occurs during a real collision. 
The tangential component of the contact force models both static and dynamic surface friction. These are calculated by placing a linear spring at the contact point, attached to both particles, at the beginning of the collision 22, 23, producing a restoring frictional force $\mathbf{f}_{t}$. The magnitude of the elongation of this tangential spring is truncated in order to satisfy the local Coulomb yield criterion $\left|\mathbf{f}_{t}\right| \leq \mu\left|\mathbf{f}_{n}\right|$. In addition, we have also implemented rolling resistance [24]. The following, though not an exact transcription, is the model as was presented by Ai et al. 225] so the the term coefficient of rolling resistance $\mu_{r}$ is defined as a dimensionless parameter:

$\mu_{r}=\tan \beta$

where $\beta$ is the angle of rolling resistance, which is the maximum angle of a slope on which the rolling resistance torque counterbalances the torque produced by gravity acting on the body. The total rolling resistance torque $M_{r}$ consists of a spring torque $M_{r_{k}}$ and a viscous damping torque $M_{r_{d}}$ in this model:

$M_{r}=M_{r_{k}}+M_{r_{d}}$

In our code, this is implemented in an incremental fashion just as suggested by Ai et al. [25]; this is:

$\Delta M_{r_{k}}=-k_{r} \Delta \theta_{r}$

For any two particles $i$ and $j$ in contact, $k_{r}=2 J_{n} R_{r} F_{n}$ is the rolling stiffness and $\Delta \theta_{r}$ is their incremental relative rotation. $J_{n}$ is a dimensionless coefficient theoretically found to be between 0.25 and 0.5 for cylinders [26]. The quantity $R_{r}=\left(r_{i} r_{j}\right) /\left(r_{i}+r_{j}\right)$ is the rolling radius of the particles in contact. For this, a winding spring provides a torque to particles in contact. In form, it is very similar to surface friction, but is instead related to the relative angular displacement. This type of friction allows us to obtain aggregates with angles of friction of up to $\sim 35^{\circ}$, typical of cohesionless aggregates on Earth, though angles of $\sim 40^{\circ}$ are not rare.

The simulation consists of a rectangular box with periodic boundary conditions and the same dimensions as in the experiments. The grains are spherical, but we have implemented a rolling friction model as suggested by $\mathrm{Ai}\left[25\right.$ such that an angle of friction of $37^{\circ}$ could be supported. The grain sizes were uniformly distributed in the range $[6.0,12.5] \mathrm{mm}$ and randomly assigned to different particles. Surface-surface friction was fixed at $\mu_{k}=0.6$ (as specified by the material supplier); graingrain collisions had a coefficient of restitution $e$ between 0.25 and 0.65 . An illustration of the simulation set-up is shown in Fig. 6.

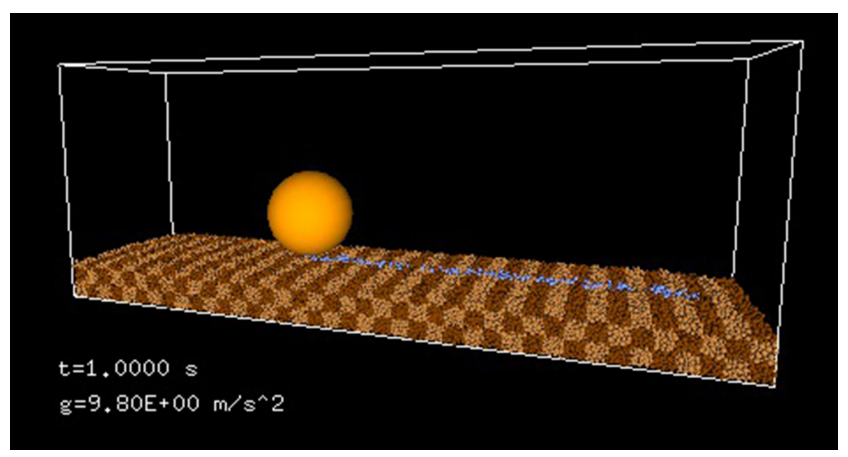

Fig. 6 Typical simulation set-up; the spherical pod has the mass of the medicine ball used in the experiments. The blue particles are those that were touched by the pod as it passed.

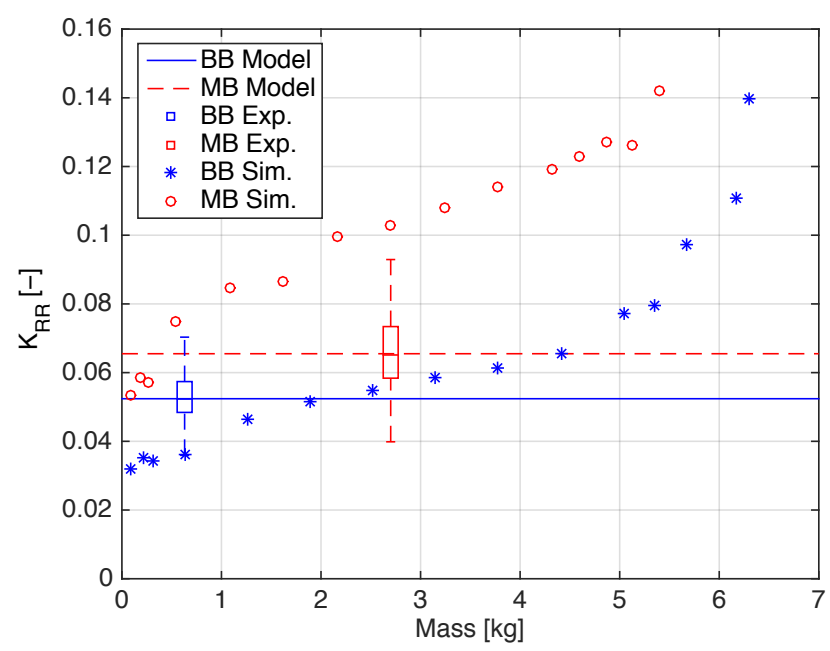

Fig. 7 Distributions of the measured $K_{r r}=j^{-1} C_{r r}$ for the experiments, simulations, and model.

In our simulations, we initially used pods of the same size, mass, and inertia as those tested in the experiments. The mean rolling resistance coefficient obtained through the simulations was $K_{r r}=0.038$ for the basketball-equivalent and $K_{r r}=0.080$ for the medicine ball-equivalent. These values differ by respectively $-27 \%$ and $+22 \%$ relative to the experimental values, and are included in Fig. 7 together with the experimental results. In other words, the simulations agree on the order of magnitude of both experimentally measured coefficients, but slightly underestimate the basketball's coefficient and slightly overestimate the medicine ball's coefficient.

To further investigate this difference, we carry out additional simulations where the masses of the two balls are varied between $0.1 \mathrm{~kg}$ and $5.0 \mathrm{~kg}$, while maintaining their respective massless inertias and sizes. The corresponding coefficients have also been included in Fig. 7. and reveal a clear mass-dependency in the rolling resistance coefficient. For the basketball $(j=2 / 3$, in blue), a sharp increase in $K_{r r}$ is observed at higher masses, 
seemingly indicative of the onset of significant plowing effects. This sharp increase is not observed for the medicine ball ( $j=2 / 5$, in red). To further investigate these trends, we review the pods' energy characteristics in the simulations.

We find that part of the pods' initial energy is transferred to the gravel as they roll, and then dissipated through friction or used to plastically deform the granular surface in a plowing process. Heavier pods are subject to more plowing and thus experience a greater resistance from the surface. This suggests that the continuous collisions between the ball and the individual grains, a second source of energy dissipation, govern the considered regime of motion. To substantiate this claim, we investigate how much energy is spent by both factors. Additionally, we are interested in the limit case of very light and very heavy balls. Given that the initial and final state of the system are known, as well as the amount of dissipated energy, it is possible to calculate a number of revelant quantities. Specifically, these are:

$\begin{cases}W_{f r}: & \text { Work done by friction } \\ \Delta E P_{g}: & \text { Potential energy change of grains } \\ \Delta E P_{b}: & \text { Potential energy change of ball } \\ \Delta E K_{b}: & \text { Kinetic energy change of ball }\end{cases}$

The amount of energy $W_{i m p}$ dissipated by the microcollisions between the ball and the grains is then found as:

$W_{i m p}=\Delta E P_{g}+\Delta E P_{b}+\Delta E K_{b}-W_{f r}$

Calculating this energy balance for our simulations, we find that balls with a low mass spend most of their energy in collisions. Both $\Delta E P_{g}$ and $W_{f r}$ increase with the mass of the ball, indicating a greater degree of plowing for heavier balls, regardless of their internal geometry. As this happens, the amount of energy that is dissipated through micro-collisions between the different balls and the regolith decreases from $90 \%$ to $60 \%$. This shows that plowing becomes important at higher masses, which was particularly visible in a sharp increase in $K_{r r}$ of the basketball for masses greater than $5 \mathrm{~kg}$.

\section{Discussion}

In experiments with a basketball and medicine ball rolling on gravel, we found that both balls left behind a shallow groove, which indicates that plowing is negligible in the considered regime of motion. This is further justified by a computation of the plowing ratio, which is much smaller than ratios encountered in previous works where plowing was found to be significant. The measured coefficient values are independent of the ball velocity, which is in agreement with literature.

When assuming a sphere to roll without plowing on a granular bed, the geometry of micro-collisions between the sphere and the bed allows the development of a simplified model for the coefficient of rolling resistance. This model predicts a coefficient independent of the velocity of the sphere, which matches the experimentally measured behavior and agrees with literature. Second, it predicts an inverse relationship of $K_{r r}$ with the reduced inertia $(1+j)$ of the considered sphere. This again agrees with the experimentally observed trends and makes intuitive sense, as the reduced inertia represents the resistance of a sphere against changes in its rolling motion. In other words, the model states that for a given granular bed and sphere size, a sphere will experience less rolling friction when its mass is concentrated in its shell, and will experience more rolling friction when its mass is concentrated at its center.

This model is also in agreement with soft-sphere DEM simulations that recreated the experiments. The simulations further allow for an investigation of the sources of energy dissipation responsible for the rolling resistance force and torque. For the tested ball masses, we find that the percentage of energy dissipated through micro-collisions varies between $90 \%$ and $60 \%$. The simulations hence reveal a mass dependency that is not captured by the analytical model. It is expected that this becomes negligible in the limit of low gravity. At higher masses, further simulations reveal a significant increase in the amount of energy dissipated through plowing.

At low masses, these simulation results are consistent with the model assumption that micro-collisions between the ball and the granular bed govern rolling friction in the considered regime of motion, and explains why our simplified model can successfully predict the order of magnitude of the coefficient of rolling resistance. In fact, the analytical model over-estimates the dissipation of energy through micro-collisions by assuming the grain is not movable, i.e. that the ball is very light. In doing so, it also neglects the energy spent in plowing. Within the conditions of our experiments, it seems these two small effects have compensated each other, and an order-of-magnitude $K_{r r}$ estimate is obtained with the analytical model, for balls with a mass lower than $3 \mathrm{~kg}$.

The estimates of rolling resistance coefficient values have a number of geophysical applications, such as analyses of the evolution of talus slopes [27. Another application of interest relates to the exploration of small bodies of the Solar system such as asteroids and 


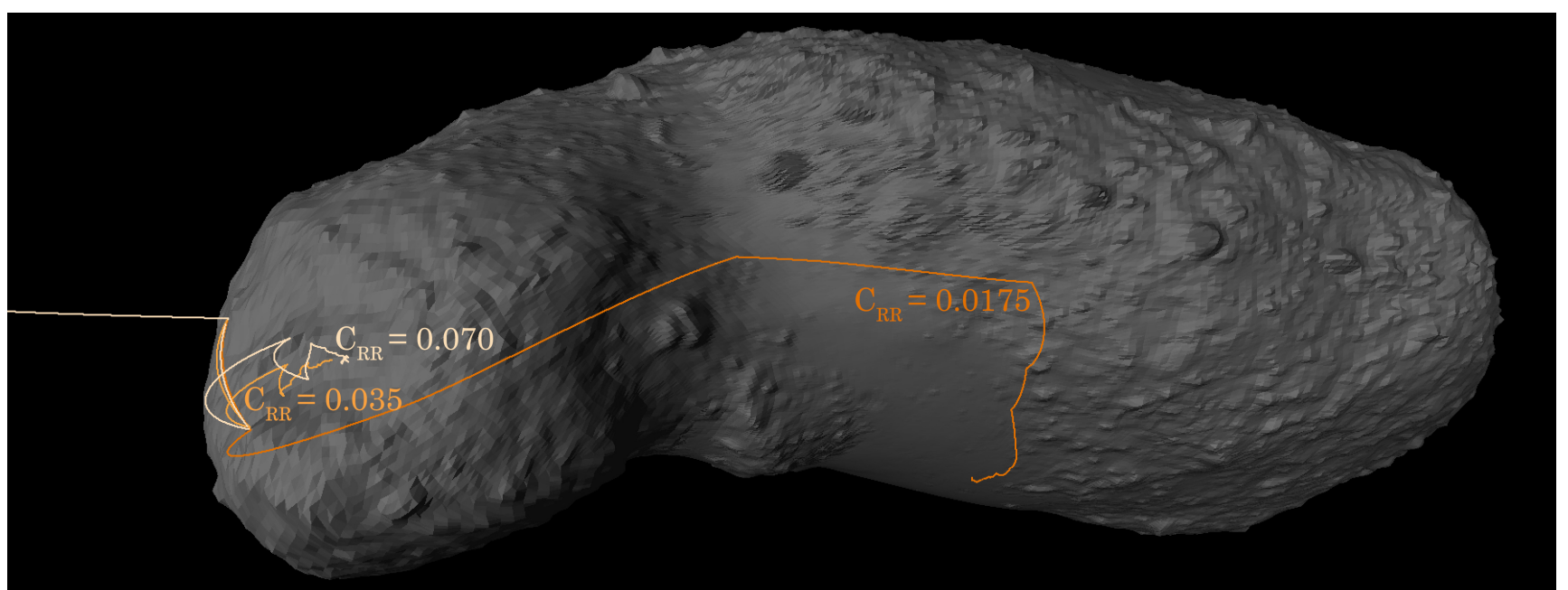

Fig. 8 Simulations of lander descent trajectories to asteroid Itokawa for various $C_{r r}$ values. The dark trajectory has a high $C_{r r}=0.0175$, the intermediate trajectory has a nominal $C_{r r}=0.035$, and the light trajectory has a low $C_{r r}=0.0705$.

comets, which are known to have loose, granular surfaces. Missions such as the proposed Binary Asteroid in-Situ Explorer (BASiX) aim to explore these bodies using uncontrolled, spherical lander packages [1]. These landers are released from orbit, balistically descend to their respective target, and operate a number of scientific instruments once settled on its surface [28,29]. As identified in [30,31,32, rolling resistance is one of two major sources of energy dissipation of the landers. It therefore affects the landers' settling time and profile, and in turn imposes mission and hardware constraints such as required battery capacity. As our model for the rolling resistance is independent of the gravitational acceleration $g$, it can be applied to the microgravity environment present on these small bodies (ignoring cohesion effects that may contribute to rolling resistance).

To illustrate the influence of rolling resistance on the descent and settling trajectory of lander, Fig. 8 shows three simulations of lander deployment to asteroid Itokawa, where different values of $C_{r r}$ were used. These simulations take into account the complex gravitational field, surface shape, and contact interactions between a lander and an asteroid; for detailed discussions the reader is referred to 33 . Rolling resistance affects the dissipation of energy during collisions and contact between a lander and the asteroid surface. Due to this difference in $C_{r r}$, the landers in the simulations have slightly different velocities following the first impact, shifting the location of the second impact. As the surface features on the asteroid have local slopes that vary strongly, the location of third impact is offset even further from the nominal trajectory. This effect propagates throughout the lander trajectory and provides a chaotically different descent profile. From a global perspective, the coefficient of rolling resistance affects the rate of energy dissipation throughout the lander trajectory, thereby controlling the time a lander takes to come to rest on the asteroid surface. An accurate estimate of $C_{r r}$ is therefore required to predict this settling time, which will influence the hardware selection.

\section{Conclusions}

In this paper, we have studied the coefficient of rolling resistance of a spherical pod rolling without slipping and without plowing on a granular bed, by means of experiment, analysis, and simulation. For this regime of motion, experiments reveal a rolling resistance coefficient independent of mass and velocity. We found mean coefficient values of $K_{r r}=0.052$ for a basketball and $K_{r r}=0.066$ for a medicine ball.

An exhaustive rolling resistance model should account for this plowing, but for the considered regime, we have constructed a simplified model that provides an approximation for the contribution of micro-collisions to rolling resistance, by ignoring plowing effects. This model predicts a rolling resistance coefficient independent of the pod velocity, matching the experimentally observed behavior. Additionally, it predicts an inverse relationship between the coefficients and the reduced inertia $(1+j)$ of the considered spheres. This too agrees with the observed trends.

Simulations further supported these coefficient values, but did reveal a mass dependency that is not captured by the model. Analysis of the sources of energy dissipation in the simulations showed that this dependency results from the plowing of the spheres into the granular bed, but that its contribution remains small for low weights. For heavy weights, plowing becomes 
the dominating source of energy dissipation. The coefficient estimates have applications to the design and dynamics of lander pods on asteroid and comets, as landers on these bodies have very low weights.

\section{Acknowledgements}

The authors would like to thank Prof. C. Hrenya at CUBoulder for allowing her laboratory space to be used for this work. ST, PS and DS acknowledge funding support from NASA Grants NNX11AQ35A, NNX10AJ66G and NNX11AP24G respectively. DDR acknowledges support from CU-Boulder and Université de Toulouse.

\section{References}

1. R. Anderson, D. Scheeres, S. Chesley, the BASiX Science Team, in 45th Lunar and Planetary Science Conference (2014). URL http://www.hou.usra.edu/meetings/ 1psc2014/pdf/1571.pdf

2. S. Tardivel, D. Scheeres, Journal of Guidance, Control, and Dynamics 36, 700 (2013). DOI 10.2514/1.59106. URL http://arc.aiaa.org/doi/abs/10.2514/1.59106

3. F.V. De Blasio, M.B. Saeter, Phys. Rev. E 79, 022301 (2009). DOI 10.1103/PhysRevE.79.022301. URL http: //link.aps.org/doi/10.1103/PhysRevE.79.022301

4. G.H. Ristow, F.X. Riguidel, D. Bideau, Journal de Physique I 4(8), 1161 (1994)

5. S. Dippel, G.G. Batrouni, D.E. Wolf, Phys. Rev. E 54, 6845 (1996). DOI 10.1103/PhysRevE.54.6845. URL http://link.aps.org/doi/10.1103/PhysRevE.54.6845

6. D.E. Wolf, F. Radjai, S. Dippel, Philosophical Magazine B 77(5), 1413 (1998)

7. M.A. Aguirre, I. Ippolito, A. Calvo, C. Henrique, D. Bideau, Powder technology 92(1), 75 (1997)

8. F.X. Riguidel, A. Hansen, D. Bideau, EPL (Europhysics Letters) 28(1), 13 (1994)

9. L. Samson, I. Ippolito, D. Bideau, G. Batrouni, Chaos: An Interdisciplinary Journal of Nonlinear Science 9(3), 639 (1999)

10. L. Samson, I. Ippolito, G. Batrouni, J. Lemaitre, The European Physical Journal B-Condensed Matter and Complex Systems 3(3), 377 (1998)

11. C. Henrique, M.A. Aguirre, A. Calvo, I. Ippolito, S. Dippel, G.G. Batrouni, D. Bideau, Physical Review E 57(4), 4743 (1998)

12. N.V. Brilliantov, T. Pöschel, EPL (Europhysics Letters) 42(5), 511 (1998)

13. A. Tan, American Journal of Physics 53(9), 811 (1985)

14. S. Van wal, D.J. Scheeres, in 26th AAS/AIAA Space Flight Mechanics Meeting (2016)

15. P. Sánchez, M.R. Swift, P.J. King, Physical review letters 93(18), 184302 (2004)

16. P.A. Cundall, in Proc. Symp. Rock Fracture (ISRM), Nancy, vol. 1 (2013), vol. 1

17. P.A. Cundall, R.D. Hart, Engineering computations 9(2), $101(1992)$

18. P. Sánchez, D.J. Scheeres, Meteoritics \& Planetary Science 49(5), 788 (2014). DOI 10.1111/maps.12293. URL http://dx.doi.org/10.1111/maps.12293
19. P. Sánchez, D.J. Scheeres, The Astrophysical Journal $\mathbf{7 2 7}(2), 120$ (2011). URL http://stacks.iop.org/ $0004-637 \mathrm{X} / 727 / \mathrm{i}=2 / \mathrm{a}=120$

20. P. Richard, A. Valance, J.F. Métayer, P. Sanchez, J. Crassous, M. Louge, R. Delannay, Physical review letters 101(24), 248002 (2008)

21. D. Tildesley, M. Allen, Clarendon, Oxford (1987)

22. H. Herrmann, S. Luding, Continuum Mechanics and Thermodynamics 10(4), 189 (1998). DOI 10.1007/ s001610050089. URL http://dx.doi.org/10.1007/ s001610050089

23. L.E. Silbert, D. Ertas, G.S. Grest, T.C. Halsey, D. Levine, S.J. Plimpton, Phys. Rev. E 64, 051302 (2001). DOI 10.1103/PhysRevE.64.051302. URL http://link.aps . org/doi/10.1103/PhysRevE.64.051302

24. P. Sánchez, D.J. Scheeres, Icarus 271, 453 (2016). DOI http://dx.doi.org/10.1016/j.icarus.2016.01.016. URL http://www.sciencedirect.com/science/article/pii/ S0019103516000208

25. J. Ai, J.F. Chen, J.M. Rotter, J.Y. Ooi, Powder Technology 206(3), 269 (2011)

26. J. Bardet, Q. Huang, Powders and grains 93, 39 (1993)

27. F.V. De Blasio, M.B. Sæter, Earth Surface Processes and Landforms (2014)

28. S. Tardivel, D.J. Scheeres, P. Michel, S. Van wal, P. Sánchez, Journal of Spacecraft and Rockets 51(6), 1857 (2014)

29. S. Tardivel, D.J. Scheeres, P. Michel, in 24th AAS/AIAA Space Flight Mechanics Meeting (2014), 14-355

30. S. Van wal, The ballistic deployment of asteroid landers. Master's thesis, Delft University of Technology (2015)

31. S. Tardivel, D.J. Scheeres, P. Michel, S. Van wal, P. Sánchez, Journal of Spacecraft and Rockets 51(6), 1857 (2014)

32. S. Tardivel, The deployment of scientific packages to asteroid surfaces. Ph.D. thesis, University of Colorado (2014)

33. S. Tardivel, D.J. Scheeres, P. Michel, S. Van wal, P. Sánchez, Journal of Spacecraft and Rockets 51(6), 1857 (2014) 